\title{
Development of a real-time test bed for indoor climate simulation in a VR environment using a digital twin
}

\author{
Kushagra Mathur ${ }^{1}$ Christoph Nytsch-Geusen ${ }^{1}$ \\ Lucas Westermann ${ }^{1}$ \\ ${ }^{1}$ Institute of Architecture and Urban Planning, \\ Berlin University of the Arts, Germany, \\ k.mathur@udk-berlin.de, nytsch@udk-berlin.de, \\ l.westermann@udk-berlin.de
}

\begin{abstract}
This paper describes the development process of a test bed for an interactive VR (virtual reality) environment for indoor climate simulations of buildings. The basic idea is to reproduce the simulated indoor climate of a thermal room model in a climate chamber with the help of air conditioning devices and thus to make the indoor climate directly physically experienceable for a user in real-time. In a first step, the real test bed is mapped with the help of a digital twin and simulated in parallel with the room model. In a second step, the digital twin is replaced by the real test bed and the Modelica room model is included then as an embedded model. In this way, the real test bed can be operated with the control algorithm which has been evaluated and optimized in a previous step. The described approach is demonstrated in a case study using a simple single-zone building model.
\end{abstract}

Keywords: hardware in the loop simulation, digital twin of a test bed, interactive virtual reality environment

\section{Introduction}

The traditional workflow in thermal building and indoor climate simulation consists of the creation of a thermal building model with simulation programs such as IDA ICE (https://www.equa.se/de/ida-ice) or EnergyPlus (https://energyplus.net), in which geometry, construction, and boundary conditions like the user behavior or outdoor climate of the real building or room are represented. As a result, the user receives time series of physical quantities describing the indoor climate, such as the air temperature, the radiation temperature, the humidity or the thermal comfort index PMV/PPD according to Fanger from these programs. In this way of working, simulation results are perceived by the user purely intellectually in the form of diagrams on a monitor.

However, since the indoor climate is primarily perceived via the physical senses, an immersive simulation process in which the user can physically experience the calculated indoor climate can lead to a deeper understanding of simulation scenarios. This approach is applied in the research project GEnEff, where the indoor climate simulated with a Modelica based thermal room model is reproduced in real-time with the help of a climate chamber and can thus be directly perceived by the user. The users are also visually immersed in the simulation process by using a VR environment, in which they can interact with the 3D representation of the room model. This includes, for example, opening windows or adapting the thermostat to different values of the set heating or cooling temperature, which influence the room climate. The feasibility of this simulation approach is described by the authors in NytschGeusen et al., 2017 as well as its first realization in a corresponding test bed with thermal feedback for users in Nytsch-Geusen et al., 2021.

In this paper, we will show how the development process could be supported using a Modelica based digital twin that first evaluates the thermal, hydraulic and functional properties of the test bed before it is realized with constructional effort.

\section{Related work}

The Modelica modeling language has been used in various domains to recreate parts of technical systems in test benches as a model utilizing hardware in the loop (HIL) simulations. For example, Winkler and Gühmann, 2006 describe a real-time Modelica model of an engine test bench in which the real engine is embedded in a virtual test environment. Schneider et al., 2015 presents the HIL model of a test bench for investigating the dynamic behavior of circulation pumps, where the environment consisting of the energy system technology and the building envelope is virtually replicated in a Modelica model. Baltzer et al. 2014 describe a HIL test bench in which a real heat exchanger device using the waste heat of a car engine was coupled with a virtual thermal Modelica model of the heated vehicle cabin.

\section{Methodology}

Figure 1 describes the two-step research approach. In the first step, the climate chamber is represented by a Modelica model as a digital twin of the test bed, which contains both its thermal envelope and the air conditioning devices used to reproduce the calculated room climate. 


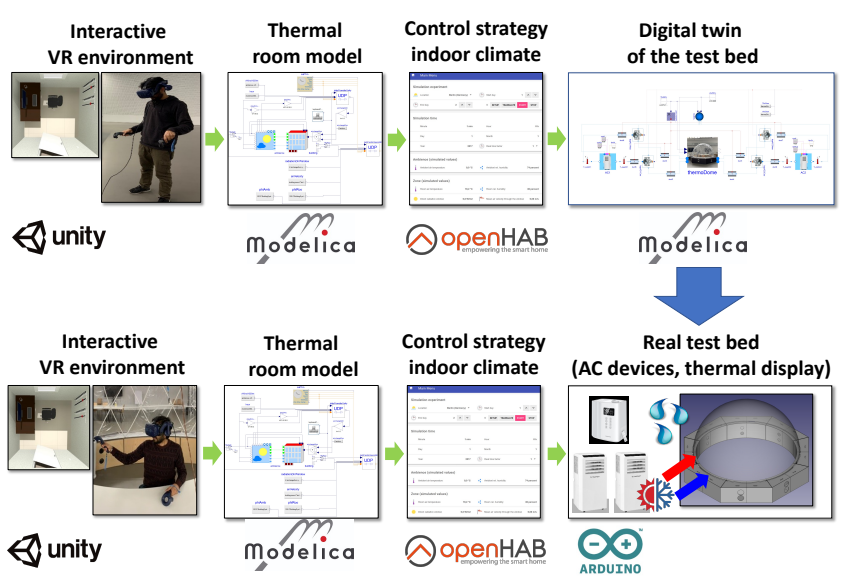

Figure 1. General research approach.

The user interacts via a VR headset in real-time with a 3D representation of the room model, which is implemented in Unity (Unity, 2021). The VR model is connected to the thermal room model also modeled in Modelica. The physical states calculated in this physical room model are sent to the event-based framework openHAB (openHAB, 2021). There, the room climate of the building model is compared with that of the climate chamber of the digital twin, and then a control algorithm is used to adjust the air conditioning devices in a manner that the differences between the two room climates are minimized. In this way, possible system configurations and control algorithms of the test bed can be evaluated in advance by making adjustments in the digital twin model. In the second step, the pre-optimized digital twin is replaced by the real test bed.

\subsection{Control system with openHAB}

openHAB (openHAB, 2021) is an open-source home automation software framework with a wide range of plugins connecting it to different hardware and software ecosystems. It uses an event-based bus to enable logical calculations or comparisons between the states of connected systems and devices as shown in Figure 2. This event bus can be manipulated and read by a REST API (Representational State Transfer Application Program Interface). For our purposes, two Python scripts access this data interface and are responsible for receiving and sending data via UDP (User Datagram Protocol) between Modelica and openHAB. This kind of data exchange was developed and evaluated by the authors in a case study for a digital twin, in which the control strategy of the building energy system for a research building was analyzed (Nytsch-Geusen et al., 2018).

Now, openHAB is used as the control system for both the test bed and its digital twin model. It performs all the logical calculations, does the comparisons of the simulated model states with the real sensor data of the climate chamber using a rule-based system. This controls the air conditioning devices of the test bed using the serial binding which then sends signals to Arduino microcontrollers. These rules are event-based logic algorithms which can perform different tasks when the conditions are triggered.

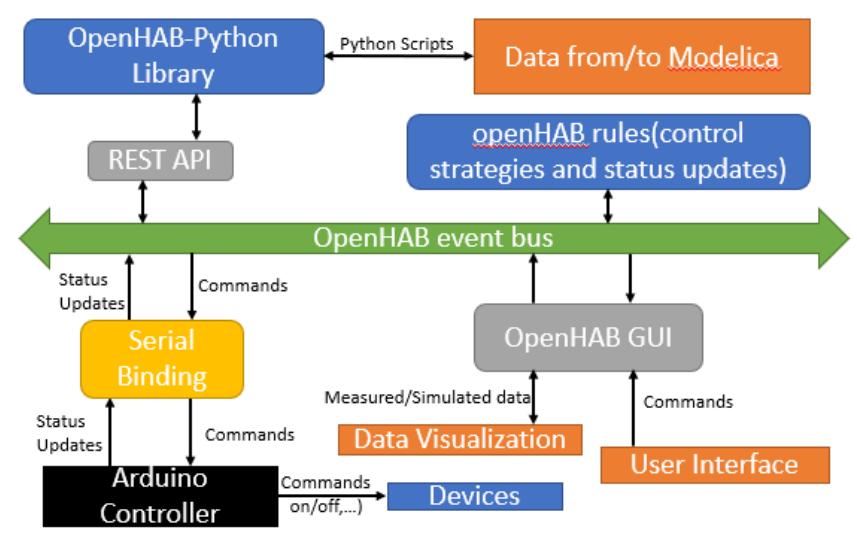

Figure 2. Interactions between openHAB event bus and connected systems and devices.

openHAB also delivers a centralized web interface to observe relevant data of the test bed and the simulation model and support the manipulation of the hardware system by the users in a standalone program without the need of receiving input data from Modelica or Unity.

\subsection{Digital twin model}

The comprehensive Modelica model of the digital twin includes different sub-models and objects interconnected with each other using distinct interfaces (compare with Figure 3). All used component models are based on or are included in the BuildingSystems library (Nytsch-Geusen et al., 2016) or the Modelica standard library (https://github.com/modelica/Modelicastand ardLibrary). This Modelica model exchanges data bidirectionally in real-time with both external software tools Unity (considering the user behavior in the VR environment) and openHAB (control logic of the climate chamber). For this purpose, a UDP data exchange model is responsible for sending or receiving data packages through UDP sockets to Unity and openHAB. This model class is implemented based on the Modelica_DeviceDrivers library (Thiele et al., 2017). It can consider also an adaptable real-time factor for increasing or decreasing the speed of the simulation experiment, depending on the present system dynamics or the presence of user interaction.

The upper part of Figure 3 shows the thermal building model for calculating the indoor climate, which receives its boundary conditions from an environment model in which different climate locations can be defined. On the other hand, the user's interactions with the visible 3D building model in the virtual reality environment are made available to the thermal building model via an interface to Unity. This can represent, for example, the user's presence in the room, opening a window, turning on the 


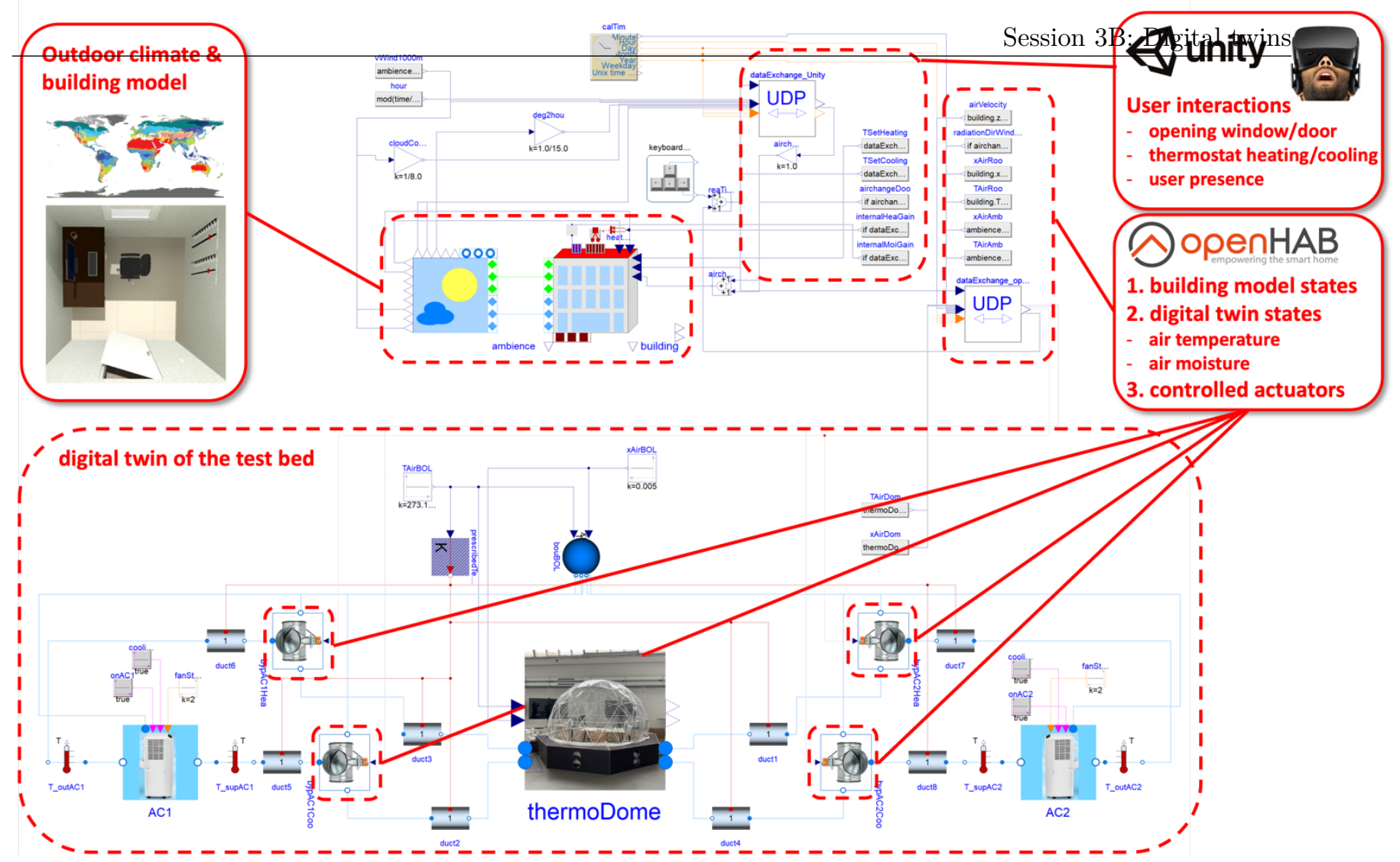

Figure 3. Digital Twin model of the real-time test bed.

lights, or changing the set values for the room thermostat. All these user interactions influence the indoor climate and are considered accordingly as dynamic boundary conditions in the thermal building model.

The lower part of Figure 3shows the sub-model of the test bed. This part of the system model was created keeping in mind the fact that this model can be used to design the real test bed. In the center, the thermal model of the climate chamber (thermoDome) is located, which is supplied with warm or cold air via a hydraulic air duct system using two air conditioning units. The hydraulic air duct system is part of the climate chamber model and it consists of two air ring tubes, one for cold air and one for warm air. Each of the air ring tubes is modeled as a detailed thermo-hydraulic model with two air inlets for the air conditioning devices and eight outlets to the inner space of the climate chamber (compare 4 with Figure 5).

Switching between hot and cold air supply is achieved via four bypass valves, which receive the control signals from the control logic of openHAB. It compares the room air temperature of the building model $\mathrm{T}_{\mathrm{Sim}}$ with the air temperature of the climate chamber model $\mathrm{T}_{\mathrm{CC}}$. This is realized via a hysteresis algorithm, using the room air temperature of the building model as the target temperature. The cooling fans supply cold air to the climate chamber if the air temperature of the climate chamber model is higher than $1 \mathrm{~K}$ above the target temperature and closes all values when the temperature of chamber is $0.4 \mathrm{~K}$ to provide a buffer for stabilising the temperature as shown in the flowchart in Figure 5. Under this threshold, both the hot and cold bypass valves are closed. Similarly, the logic opens the heating valves if the threshold of $1 \mathrm{~K}$ below the room air temperature of the building model is reached.

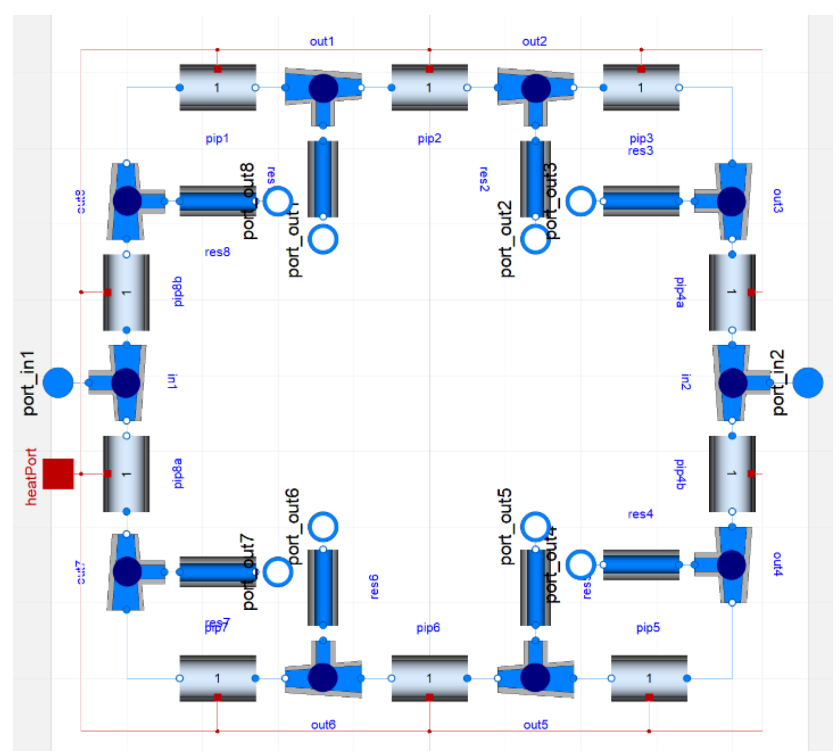

Figure 4. Thermo-hydraulic model of one of the two ring tubes which supply the climate chamber with warm or cold air through eight openings.

\subsection{Real-time test bed}

After simulating and testing the digital twin model, the real-time test bed was designed and built. In this context, more adaptations were made in the digital twin model and the test bed depending on the available hardware and their functioning. 


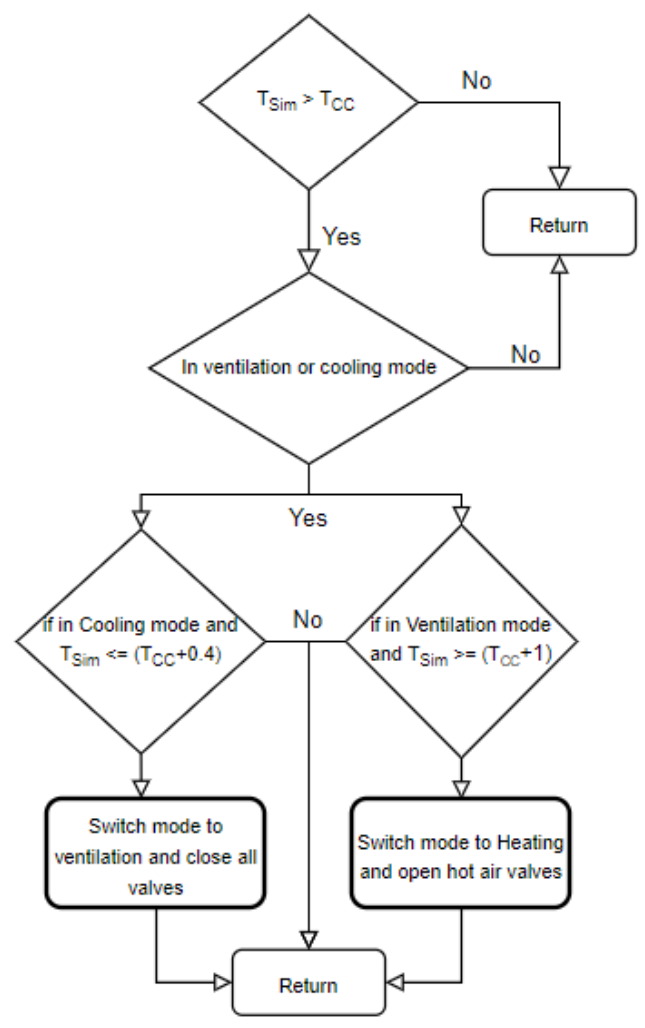

Figure 5. A flowchart depicting one of the control algorithm used for checking and changing mode of the device.

The real-time test bed consists of an igloo-shaped tent and a base level construction (see Figure 4). The inner height of the dome is $2.8 \mathrm{~m}$, and it has an inner diameter of $3.6 \mathrm{~m}$. Two air duct ring tubes in the base level construction supply the inner space of the dome with warm and cold air through 16 circular openings from two air conditioning (AC) devices (compare with Figure 4 and Figure 7). The test bed includes two air conditioners for supplying the dome. These devices have a cooling capacity of $2,050 \mathrm{~W}$, a heating capacity of $1,800 \mathrm{~W}$, and a max. air volume flow rate of $320 \mathrm{~m}^{3} / \mathrm{h}$. In addition, two additional convective tower heaters can support a fast increase of the air temperature with a max. common capacity of $4,400 \mathrm{~W}$. Two humidifiers can increase the air moisture level in the dome. The felt air movement through windows and doors can be simulated by two fans with 26 stages (max. airspeed $2.7 \mathrm{~m} / \mathrm{s}$ ). Finally, the felt solar radiation through closed or opened windows is simulated by an electric heating radiator in three stages with $850 \mathrm{~W}$, $1,650 \mathrm{~W}$, and $2,500 \mathrm{~W}$.

All the hardware can be operated by infrared remote control. These infrared signals were decoded using the irlib2 library (https://github.com/cyborg5/IRLib2) for Arduino and the same library was used to encode it. Hence, these devices were controlled by an Arduino program. Since single-hose air-conditioning devices are used, it gives the advantage that, if the device is running in cooling mode supplying cold air, the exhaust port will supply warm air.

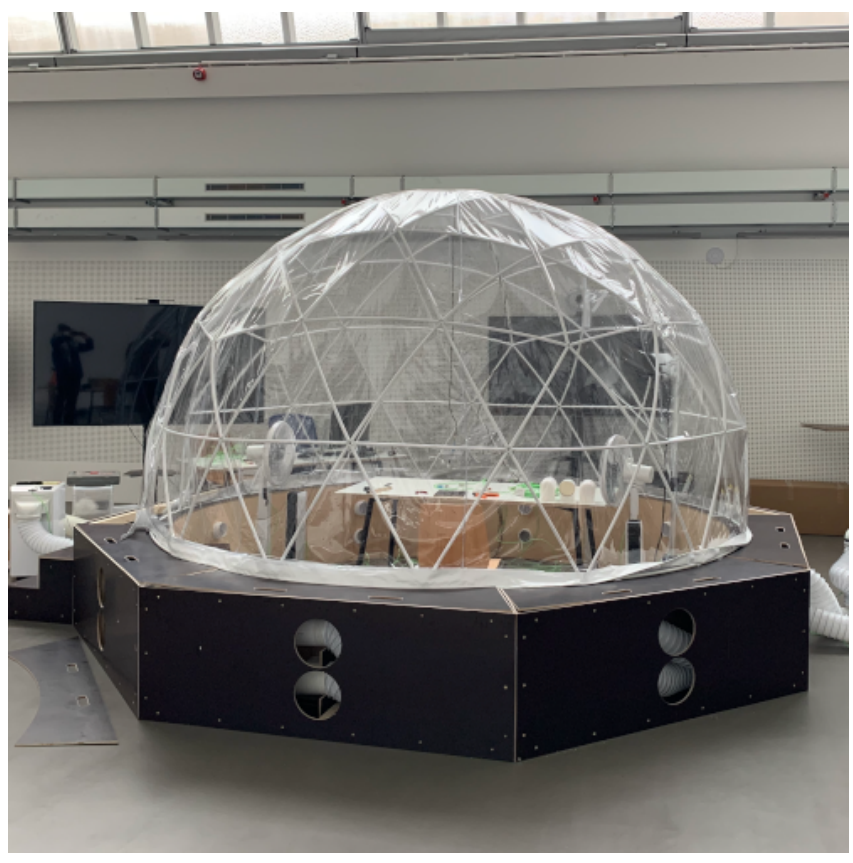

Figure 4. Real-time best bed with igloo-shaped climate chamber.

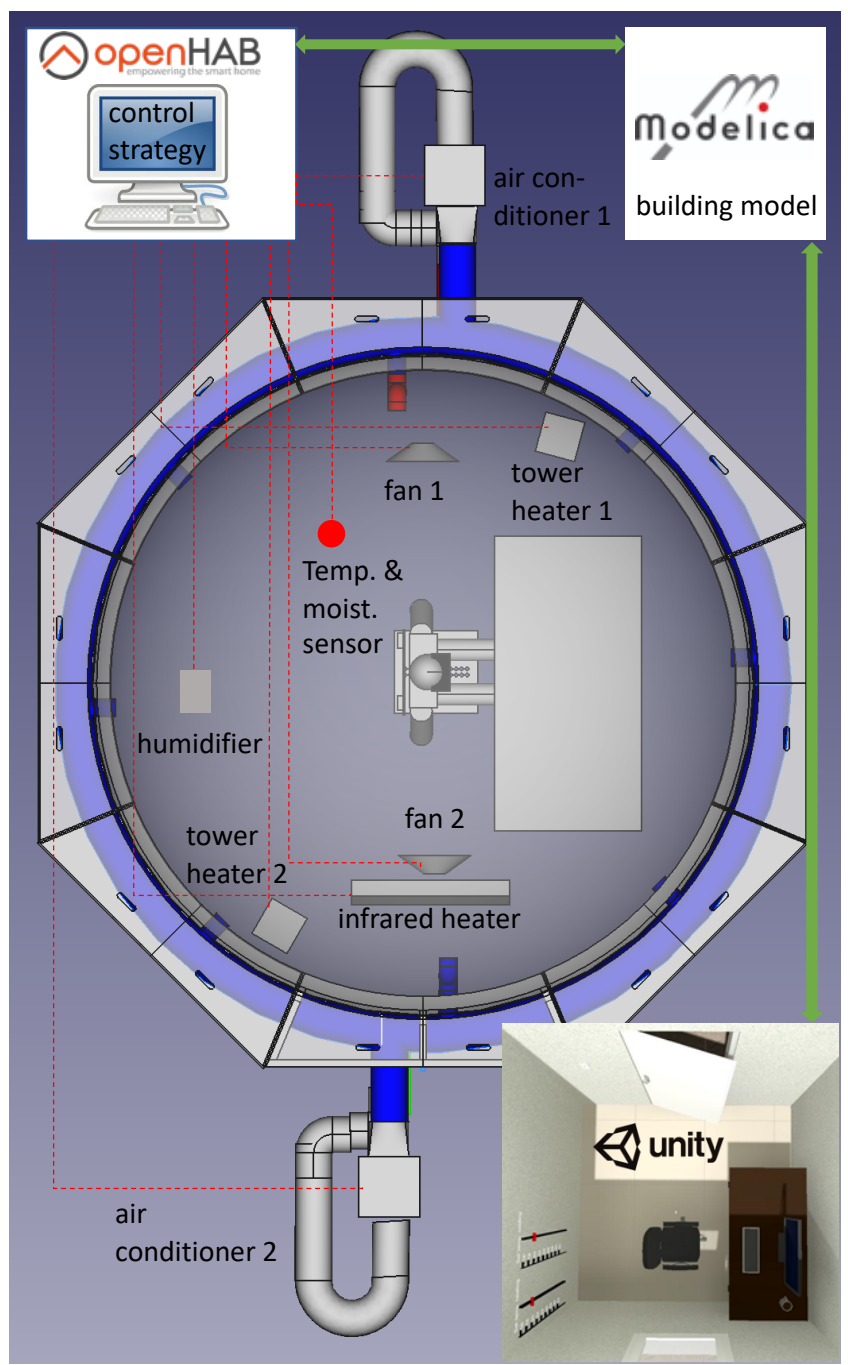

Figure 5. Configuration of the real-time test bed. 
Because of this advantage, the device does not have to switch from heating to cooling mode which takes up to ten minutes. For that purpose, bypass valves were used to switch the airflow from the AC devices. The valves were also controlled by an Arduino using relays. The Arduino receives commands from openHAB through the serial port. Reusing the same rules as for the digital twin of the test bed, the air temperature inside the climate chamber can be manipulated.

\subsection{Embedded building model}

Figure 6 shows the Modelica thermal building model embedded in the test bed. In this case, it includes only the thermal room model and the UDP interface models to Unity and openHAB. It will be running in parallel to the real-time usage of the climate chamber and provides the necessary data for controlling and manipulating the indoor climate (air temperature, air moisture, air velocity through open windows, solar radiation through transparent façade areas, ...) inside of the igloo. Certain parameters in the Modelica model can be kept dynamic, which can change with time and simulation will adapt accordingly. Such parameters include the location of the user (e.g., presence or absence in the room), the window opening state, or the set temperatures for heating and cooling.

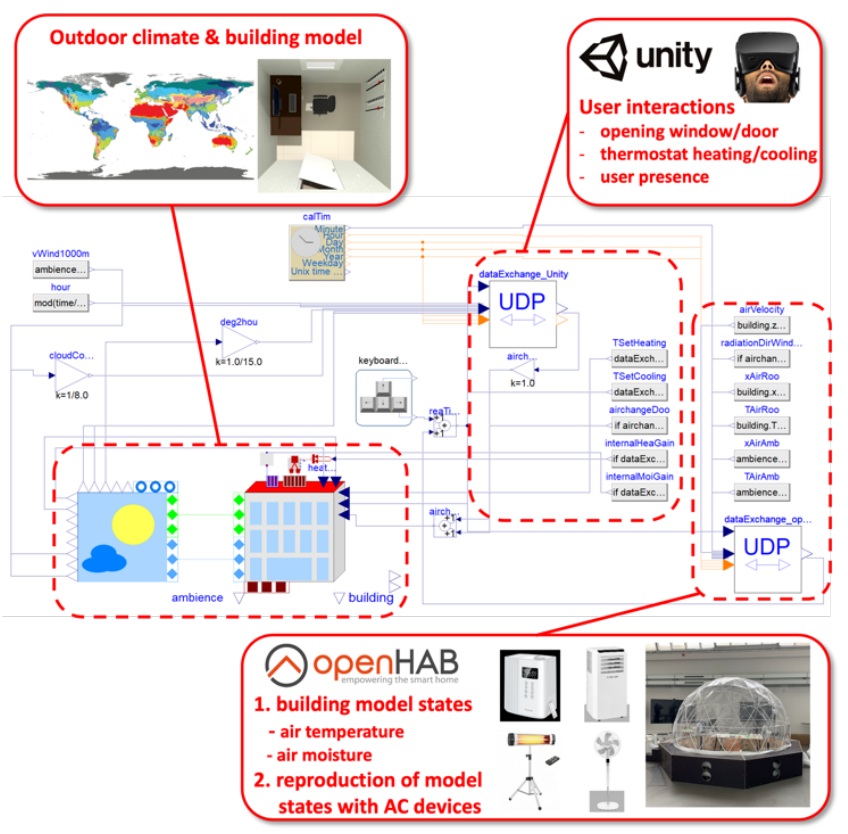

Figure 6. Embedded Modelica thermal building model.

\section{Case study}

The case study demonstrates the two-step research approach with the Modelica digital twin model and the Modelica HIL model. The simulation scenario takes place on the $1^{\text {st }}$ of January at the climate location in El Gouna (Egypt) over a real-time period of 4,600 seconds. A simple cubic shaped one zone building model is used with a dimension of $3 \mathrm{~m} \mathrm{x} 3 \mathrm{~m} \mathrm{x} 3 \mathrm{~m}$. It consists of four $20 \mathrm{~cm}$ concrete walls with outer $(1.5 \mathrm{~cm})$ and inner $(2 \mathrm{~cm})$ plaster, a $3 \mathrm{~cm}$ thick door, and a window with a U-value of $3.0 \mathrm{~W} / \mathrm{m}^{2} \mathrm{~K}$ and g-value of 0.8 . The floor and roof are constructed from $30 \mathrm{~cm}$ thick concrete. For adapting the indoor climate, the building model is equipped with an ideal heater and an ideal cooler, each with a max. capacity of $2,000 \mathrm{~W}$. The set temperature for heating and cooling can vary in a range between $16^{\circ} \mathrm{C}$ and $32^{\circ} \mathrm{C}$.

The scenario starts with an initialization temperature for the room model of $24^{\circ} \mathrm{C}$, both for the enclosed room air and for all component layers of the wall, floor, and ceiling models. Initially, a set cooling temperature of $32^{\circ} \mathrm{C}$ and a set heating temperature of $18^{\circ} \mathrm{C}$ are defined by the user in the VR environment, so that neither heating nor cooling is required in the room in the beginning of the experiment. The ambient temperature of the room model is approx. $20{ }^{\circ} \mathrm{C}$ during the experiment with a slight downward trend. After 20 minutes, the set cooling temperature is reduced from $24^{\circ} \mathrm{C}$ to $20^{\circ} \mathrm{C}$ by the user. From the 30 th minute, the set heating temperature is then raised in several $2{ }^{\circ} \mathrm{C}$ steps every 10 minutes until it reaches 32 ${ }^{\circ} \mathrm{C}$ (for the digital twin) or $30{ }^{\circ} \mathrm{C}$ (for the real test bed) at the end of the experiment. The set cooling temperature is raised to $32{ }^{\circ} \mathrm{C}$ during the heating phase to avoid simultaneous heating and cooling in the building model.

\section{Results}

\subsection{Digital twin model}

Figure 7 shows the dynamic behavior of the thermal building model and the indoor climate of the climate chamber model. It can be seen that the calculated air temperature of the indoor model in the climate chamber can be reproduced well with the help of both air conditioning devices and the control strategy for the bypass dampers implemented in openHAB. The ambient temperature of the climate chamber model was set to $24{ }^{\circ} \mathrm{C}$, corresponding to the ambient conditions of the laboratory in which the real test bed is located. The peaks of cooling and heating power are visible, which are caused by the user due to the change of the set temperatures.

\subsection{Real-Time test bed}

Figure 8 above shows the simulated indoor air temperature of the thermal building model and its replication in the real climate chamber. In this case, the calculated air temperature can be replicated less well in the climate chamber than with its digital twin over the entire period of the experiment. Qualitatively, the indoor climate is correctly reproduced, but the real climate chamber has difficulties in reproducing fast dynamic changes of the air temperature. Overall, the multi-stage heating-up process is better reproduced than the coolingdown process. The number of heating and cooling periods in the real experiment is significantly lower but they durations significant longer compared to the experiment with the digital twin. 

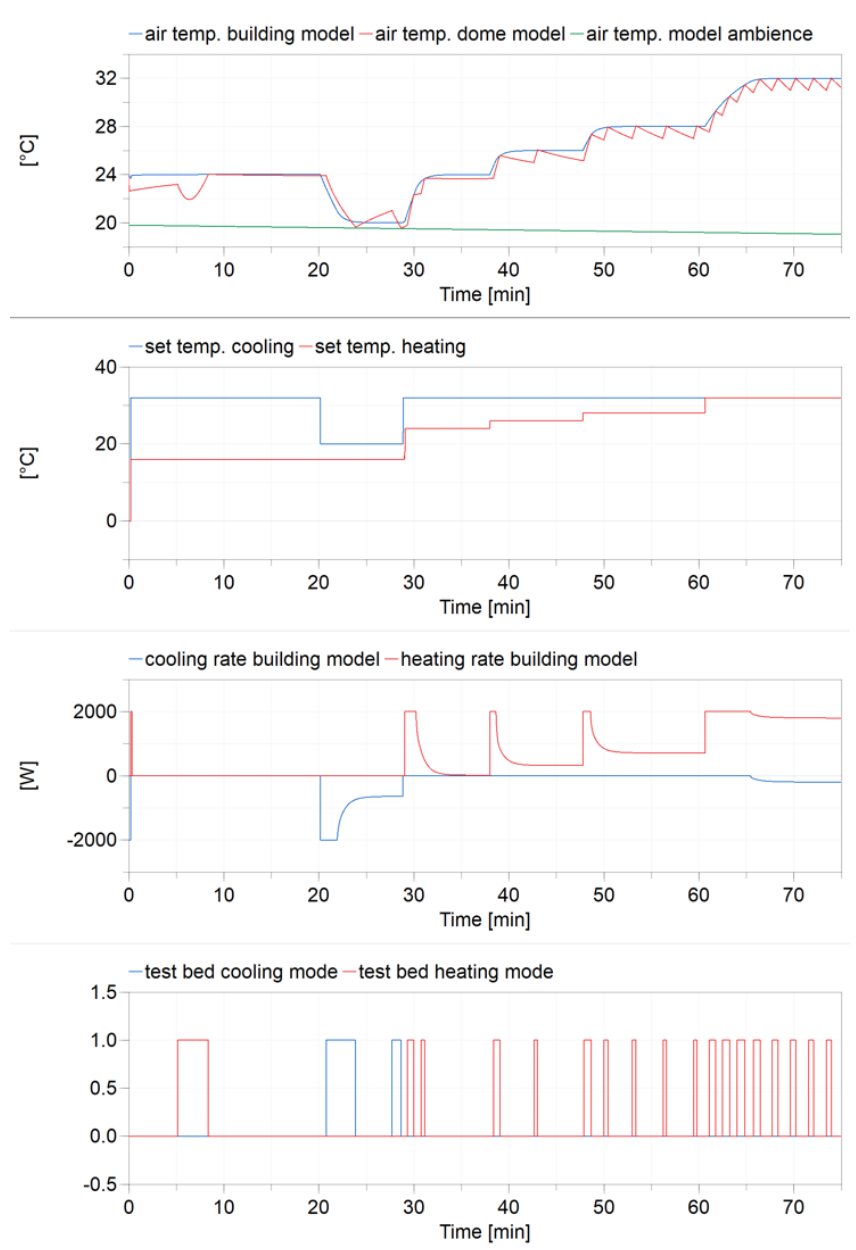

Figure 7. Results for the digital twin model.

\section{Discussion}

The case study has shown that the design of the test bed and the evaluation of associated control strategies can be tested in advance with the use of a digital twin. This knowledge can then be profitably transferred to the real test bed. It seems important to increase the cooling and the heating capacity in the climatic chamber to be able to simulate fast changes in the indoor climate sufficiently well in the real climatic chamber. This also includes a further development of the present discrete control strategy for the heating and cooling case, which has been kept very simple up to now. The use of continuous controllers or model-based controllers can be expected to provide better control quality. Also, a model extension of the digital twin seems to be necessary, which is partially still modeled too idealized. For example, performancereducing leakages in the air distribution system are neglected, which cannot be completely prevented in the real test bed.

\section{Summary and outlook}

A two-step method is described and demonstrated in a case study to effectively support the development process of an interactive virtual reality environment for real-time simulation of indoor climate.
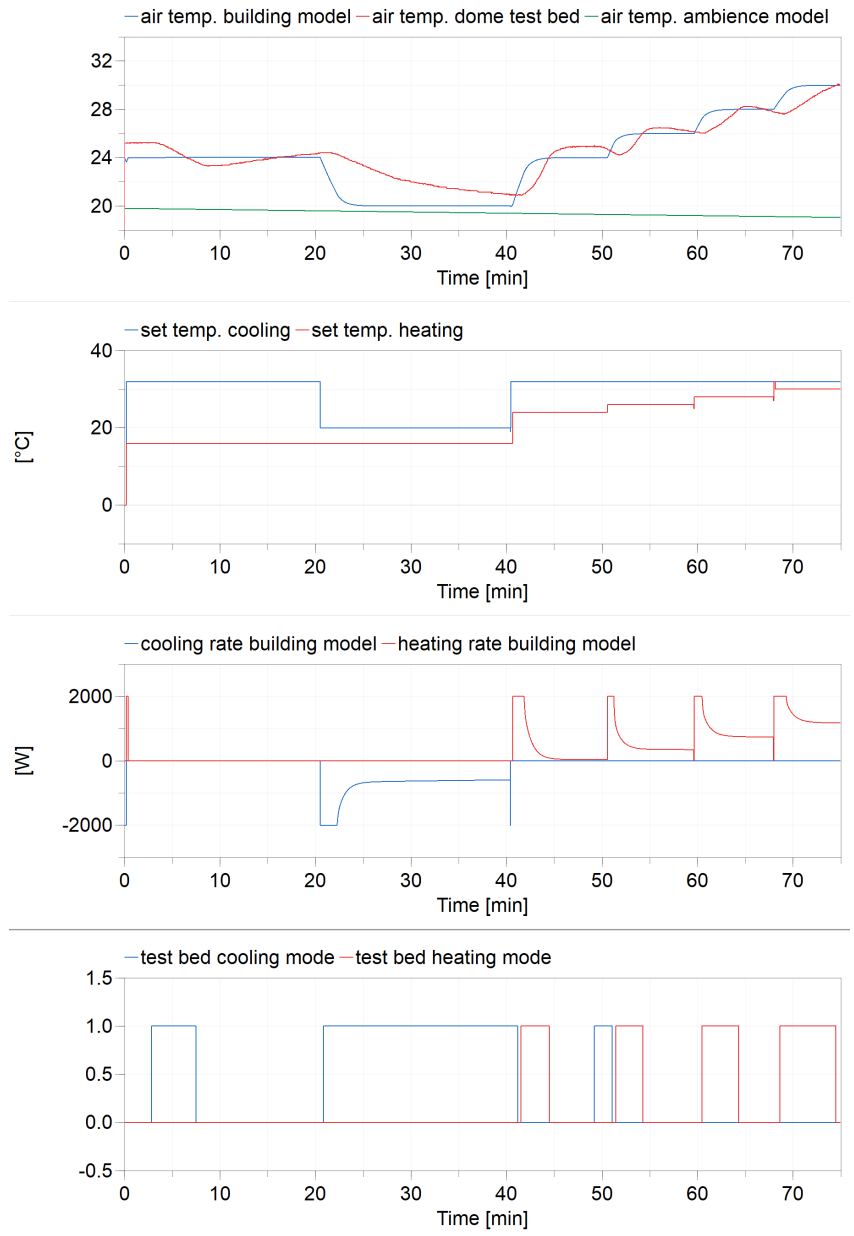

Figure 8. Results for the real-time test bed with the embedded building model.

With the help of a digital twin of the real test bed, weak points in the experimental setup can be figured out in the simulation analysis. Furthermore, control algorithms can be tested and optimized efficiently in time by using an adaptive real-time factor.

The next development steps for the climate chamber will focus on the improvement of the achievable system dynamics. For this purpose, the power for heating and cooling has to be significantly increased and the control algorithms of the climate chamber has to be further developed.

\section{Acknowledgments}

The described research was conducted within the project "EnOB: GEnEff - Neuartige Bewertung der GebäudeEnergie-Effizienz und innovative Demonstration mittels Simulationsmethoden und Virtual Reality" and funded by the Federal Ministry for Economic Affairs and Energy in Germany (reference: 03EN1017A and 03EN1017B). 


\section{References}

Baltzer, Sidney, Lichius, Thomas, Gissing, Jörg, Jeck, Peter, Eckstein, Lutz (2014). Hardware-in-the-Loop (HIL) Simulation with Modelica - A Design Tool for Thermal Management Systems. Proceedings from 10th International Modelica Conference. Lund (Sweden), 10-12 September 2014.

Nytsch-Geusen, Christoph, Banhardt, Christoph., Inderfurth. Alexander, Mucha, Katharina, Möckel, Jens, Rädler, Jörg, Thorade, Matthis and Tugores, Carles (2016). BuildingSystems - Eine modular hierarchische ModellBibliothek zur energetischen Gebäude- und Anlagensimulation. Proceedings from Conference BAUSIM 2016. Dresden (Germany), 14-16 September 2016.

Nytsch-Geusen, Christoph, Ayubi, Thaeba, Möckel, Jens, Rädler, Jörg and Thorade, Matthis (2017). BuildingSystems_VR - A new approach for immersive and interactive building energy simulation. Proceedings from Building Simulation 2017. San Francisco (USA), 7-9 August 2017.

Nytsch-Geusen, Christoph, Kaul, Werner, Kharraz, Sina (2018). Der digitale Zwilling in der energetischen Gebäude-und Anlagensimulation. Conference Proceedings from BAUSIM 2018. Karlsruhe (Germany), 26.-28. September 2018.

Nytsch-Geusen, Christoph and Mathur, Kushagra (2020). Entwicklung einer Virtual Reality-Umgebung zur interaktiven thermischen Raumsimulation. Bauphysik 42(6), 315-325.

Nytsch-Geusen, Christoph, Kaul, Werner, Mathur, Kushagra, Westermann, Lucas and Kriegel, Martin (2021). Development of an interactive virtual reality simulation environment with a thermal feedback for the user. Proceedings from Building Simulation 2021. Bruges (Belgium), 1-3 September 2021.

OpenHAB (2021). Official web site of OpenHAB: https://openhab.org (last access on 2021 May).

Schneider, Georg Ferdinand, Oppermann, Jens, Constantin, Ana, Streblow, Rita, Müller, Dirk (2015). Hardware-in-theLoop-Simulation of a Building Energy and Control System to Investigate Circulating Pump Control Using Modelica. Proceedings from $11^{\text {th }}$ International Modelica Conference. Versailles (France), 21-23 September 2015.

Thiele, Bernhard, Beutlich, Thomas, Waurich, Volker, Sjölund, Martin and Bellmann, Tobias (2017). Towards a StandardConform, Platform-Generic and Feature-Rich Modelica Device Drivers Library. Proceedings from 12th International Modelica Conference. Prague (Tschechien), 15-17 May 2017.

Unity (2021). Official web site of Unity: https://unity3d.com (last access on 2021 May).

Winkler, Dietmar and Gühmann, Clemens (2006). Synchronising a Modelica Real-Time Simulation Model with a Highly Dynamic Engine Test-Bench System. Proceedings from 4th International Modelica Conference. Vienna (Austria), 4-5 September 2006. 